\title{
Covariance Based Spectrum Sensing with Studentized Extreme Eigenvalue
}

\author{
Cebrail ÇIFLIKLI, Fatih Yavuz ILGIN
}

\begin{abstract}
The eigenvalue based detection is a low-cost spectrum sensing method that detects the presence of primary user signal at a desired frequency. In this study, the largest eigenvalue distribution used in eigenvalue based detection methods is expressed using a new centering and scaling coefficients adjustment. Thus, the detection probability $\left(P_{d}\right)$ and false detection probability $\left(P_{f a}\right)$ equations for the maximum-minimum eigenvalue (MME), maximum eigenvalue to trace (MET) and maximum eigenvalue-geometric mean (ME-GM) have been obtained again. Weibull fading channels are the best model for wireless communication. For this reason, the studies were simulated in Weibull fading channels and analysed in detail with receiver operating characteristic curves (ROC). The results were compared with traditional methods and found to be more accurate.
\end{abstract}

Keywords: blind spectrum sensing; eigenvalue based spectrum sensing; Tracy-Widom distribution; Weibull fading

\section{INTRODUCTION}

Together with increasing technological developments in recent years, there has been a considerable increase in the size of data transmitted in wireless communication. As a consequence of the increased data size, it has become necessary to use the spectrum more efficiently [1]. Cognitive Radio (CR) has become a subject that has been studied over the last years as a solution to the intense use of spectrum and the accompanying inadequacy of spectrum. CR is a new generation radio type that can identify the spectrum holes and use these holes as opportunistical. CR users intend to use the remaining spectrum from licensed users by continuously scanning the entire spectrum or a specific section of the spectrum [2-6].

Different methods such as matched filter [7, 8], energy detection $[9,10]$, cyclostationary detection $[11-$ 14], eigenvalue detection [15-17], and covariance-based detection [18] are proposed for spectrum sensing in the literature. These methods have their own advantages and disadvantages. For example, in the matched filter method, it is necessary to know the primary user signal exactly. In the cyclostationary detection method, it has a high computational cost as the sign of the cyclix of the primary user signal must be known completely. Although the energy detection method is quite simple in terms of the cost calculation, there are practical difficulties as the noise variance cannot be precisely estimated. The most significant advantage of covariance based detection over other methods is that it requires no prior knowledge (variance, modulation, channel information) about the noise signal and the primary user signal; besides, the computation cost is very low. In literature, methods such as maximum-minimum eigenvalue (MME), maximum eigenvalue-geometric mean (ME-GM), maximum eigenvalue to trace (MET), arithmetic geometric mean (A-GM), energy-minimum eigenvalue (EME) and spectrum sensing using weighted covariance matrix (WCD) have been proposed for covariance-based detection [19]. What is important in these methods is to express the parameters specified for the test statistic (TS) and the threshold value with an appropriate/realistic function. In MME, MET and ME-GM methods, the probability distribution of the largest eigenvalue of the sample covariance matrix is used. Expressing this distribution with the most realistic function is the most effective factor in the performance of the method.

In this study, it is aimed to develop eigenvalue based detection methods. Therefore, a new centering and scale adjustment is used for the largest eigenvalue distribution of Wishart matrices. In this way, the detection probability $\left(P_{d}\right)$ and false detection probability $\left(P_{f a}\right)$ equations for MME, MET and ME-GM based detection methods have been obtained again. The obtained results have been simulated in the weibull fading channel and its results have been interpreted.

The remainder of this paper is organized as follows. In section II, on the Tracy-Widom approximation of studentized extreme eigenvalues of Wishart matrices are presented. The signal model of a cognitive user with multiple antennas in Weibull fading channels is given in Section III. The covariance based spectrum sensing with studentized extreme eigenvalue detector for MME, MET and ME-GM is proposed in Section IV, and simulation results are presented in Section V. Section VI is devoted to the discussion. Finally, in Section VII, some concluding remarks are made.

In this paper, while the bold letters represent the matrix and the normal letters represent the vectors. $(\cdot)^{\prime}$, $\operatorname{var}(\cdot)$ and $\mathrm{E}(\cdot)$ indicates transpose, variance and the mathematical expectation respectively.

\section{TRACY-WIDOM APPROXIMATION OF STUDENTIZED EXTREME EIGENVALUES OF WISHART MATRICES}

The sample covariance matrix eigenvalues and the probability distribution functions of these eigenvalues are among the common topics of the communication systems besides multivariate statistic. $\boldsymbol{X}$ is an independent and identically distributed random matrix (i.i.d.) in $n \times p$ dimension; if any $\boldsymbol{W}$ matrix can be written as $=\boldsymbol{X} \boldsymbol{X}^{\prime}, \boldsymbol{W}$ matrix is called Wishart matrix where the matrix $\boldsymbol{X}$ can be complex or real in size and $\boldsymbol{X}^{\prime}$ refers to its transpose.

Let the $\mu$ and $\boldsymbol{\Sigma}$ be the mean of $\boldsymbol{X}$ matrix and covariance matrix, respectively. When $\mu=0$, covariance matrix is defined as $\boldsymbol{\Sigma}=\boldsymbol{W}=\boldsymbol{X} \boldsymbol{X}^{\prime}$. Let $\lambda_{p}$ and $\lambda_{1}$ be the largest eigenvalue and smallest eigenvalue of $\boldsymbol{\Sigma}$, respectively. The probability distribution of the statistic 
$L_{j}$ converges to the Tracy-Widom distribution of order 1 in $(n, p \rightarrow \infty)$ conditions[19].

$L_{j}=\left(\frac{\lambda_{p}(A(n))-\widetilde{\mu}_{n, p}}{\widetilde{\sigma}_{n, p}}\right) \stackrel{D}{\rightarrow} F_{1}$

where $\tilde{\mu}_{n, p}$ and $\tilde{\sigma}_{n, p}$ denotes the variance of the distribution and the center of the distribution respectively. $\tilde{\mu}_{n, p}=n^{-1}(\sqrt{n-1}+\sqrt{p})^{2}$ and $\tilde{\sigma}_{n, p}=\left(\tilde{\mu}_{n, p} /\right.$

$n)^{1 / 2}\left(\frac{1}{\sqrt{n-1}}+\frac{1}{\sqrt{p}}\right)^{1 / 3}$ is defined and $F_{1}$ represents TracyWidom distribution of order 1 [20]. $\sigma^{2}$ indicates $\operatorname{var}(\boldsymbol{X})$ and $\boldsymbol{A}(n)$ denotes normalization coefficient and is defined as $\frac{n}{\sigma^{2}} \boldsymbol{\Sigma}$. Ma, in his studies changed center $\tilde{\mu}_{n, p}$ and scale $\tilde{\sigma}_{n, p}$ coefficients as $\mu_{n, p}=n^{-1}(\sqrt{n-1 / 2}+\sqrt{p-1 / 2})^{2}$ and $\sigma_{n, p}=\left(\mu_{n, p} / n\right)^{1 / 2}\left(\frac{1}{\sqrt{n-1 / 2}}+\frac{1}{\sqrt{p-1 / 2}}\right)^{1 / 3}$ and along with this change proved that equation 1 is closer to the Tracy-Widom distribution of order 1 in finite sample lengths [20]. In addition to this study, there are a lot of studies in the literature about largest eigenvalue distribution of the covariance matrix $[6,15,21]$. However the best solution is done by using the studentized extreme eigenvalues distribution and it is defined as

$L_{c}=\left(\frac{\lambda_{p}(A(n))-\mu_{n, p, 0}}{\sigma_{c}}\right) \stackrel{D}{\rightarrow} F_{1}$.

where $\mu_{n, p, 0}, \sigma_{c}$ and $\sigma_{n, p, 0}$ is defined $\mu_{n, p}+a \sigma_{n, p}$, $\frac{n p}{2+n p}\left(\sigma_{n, p, 0}^{2}-\left(\frac{2}{n p}\right) \mu_{n, p, 0}^{2}\right)$ and $b \sigma_{n, p}$ respectively. $a$ and $b$ is fixed $E(W)=-1.206548$ and $\sqrt{\operatorname{var}(W)}=1.267941$ respectively [22].

To test the correctness of these distributions, a Gaussian random $\boldsymbol{X}$ matrix (i.i.d.) is used in the size of $6 \times 1000$ with zero mean. For the eigenvalues of the covariance matrix of the matrix $\boldsymbol{X}, L_{c}, L_{j}$ with 1000 repetition number and Tracy-Widom distribution of order 1 is shown in Fig. 1. In view of the Fig. $1, L_{c}$ statistics represents a significant improvement compared to the $L_{j}$ statistic and it seems that it is very close to the TracyWidom distribution of order 1 .

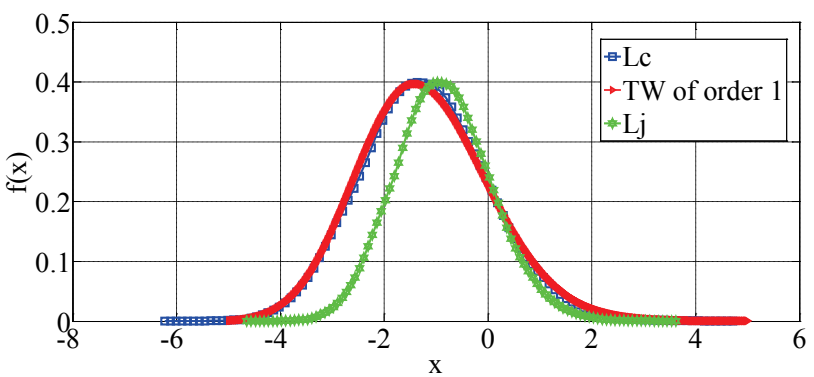

Figure $1 L_{c}, L_{j}$ statistics and TW distribution of order 1

The false alarm probability and probability of detection in spectrum sensing applications is determined/allowed by the FFC. For this reason, to describe the eigenvalue distribution with the most accurate closed function is very important in the performance of the method.
Since the relevant channel is used opportunistically in the spectrum sensing, it is very important to be able to detect the presence of the signal at the least number of samples or as soon as possible. For this reason, variance deviation for matrices of different sizes is shown graphically in Fig. 2 to see the success of the proposed method over the asymptotic approach. Variance error is indicated by $\sigma_{e}$.

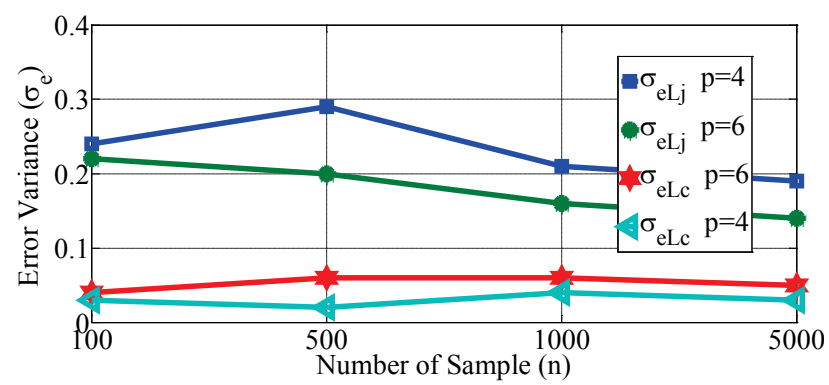

Figure 2 Variance errors by sample length for $p=4$ and $p=6$

Where the $y$-axis indicates the error deviation of the probability distribution function of the largest eigenvalue. $\sigma_{e}$ has been calculated as $\sigma_{e(L j)}=\left|\sigma_{F 1}-\sigma_{L j}\right|$ ve $\sigma_{e(L c)}=$ $\left|\sigma_{F 1}-\sigma_{L C}\right|$ to see how much the corresponding statistics deviates from the Tracy-Widom distribution of order 1 . When the graph is analysed, it is seen that the corrected variance has a much smaller margin of error than the $L_{j}$ statistics, especially in small sample numbers.

\section{SYSTEM MODEL}

In the proposed system we are trying to detect the primary user's presence through the $p$ number receiver antenna in the secondary user. $H_{0}$ indicates no primary user signal, that is, there is only noise at the corresponding frequency, and $H_{1}$ indicates the primary user signal exists. $\boldsymbol{x}(n)$ denotes the signal that the secondary user has received through $p$ number antennas and its mathematical expression is defined as

$\boldsymbol{x}(n)=\mathbb{H} \boldsymbol{s}(n) \mathbb{H}^{\dagger}+\boldsymbol{\eta}(n)$

where $\mathbb{H}$ is matrix that indicates channel response. Signals received on the secondary user under both hypotheses are as

$H_{0}: x_{i}(n)=\eta_{i}(n), \quad i=1,2, \ldots, p$

$H_{1}: x_{i}(n)=s_{i}(n)+\eta_{i}(n), \quad i=1,2, \ldots, p$

Where $x_{i}(n)$ denotes the signal that cognitive user detects from $i$ antenna and is defined as $x_{i}(n) \sim C N\left(0, \sigma_{x}^{2}\right) . \eta_{i}(n)$ is an independent and uniformly distributed complex Gaussian, and is shown as $\eta_{i}(n) \sim C N\left(0, \sigma_{\eta}^{2}\right)$. Thus, statistical covariance matrices of the received signal for $H_{0}$ and $H_{1}$ conditions are described as

$\boldsymbol{R}_{\boldsymbol{\eta}}=E\left(\boldsymbol{\eta}(n) \boldsymbol{\eta}(n)^{\dagger}\right)$

$\boldsymbol{R}_{\boldsymbol{x}}=E\left(\boldsymbol{x}(n) \boldsymbol{x}(n)^{\dagger}\right)$

Probability of false alarm $\left(P_{f a}\right)$ and Probability of detection $\left(P_{d}\right)$ equations are represented by conditional 
probability conditions $P\left(T S_{\tau}>\gamma_{\tau} \mid H_{0}\right)$ and $P\left(T S_{\tau}>\right.$ $\left.\gamma_{\tau} \mid H_{1}\right)$ respectively $[15,16]$, where, $T S_{\tau}, \gamma_{\tau}$ indicate the test statistic and threshold value of the relevant statistic. $\tau$ is used as a sub-index indicating the method.

\section{DERIVATION OF $P_{f a}$ AND THRESHOLD FOR MME}

$\lambda_{1}, \lambda_{2}, \ldots, \lambda_{p}$ and $\rho_{1}, \rho_{2}, \ldots, \rho_{p}$, are the eigenvalues of $\boldsymbol{R}_{\boldsymbol{\eta}}$ and $\boldsymbol{R}_{\boldsymbol{x}}$ respectively. Under the hypothesis $H_{0}$, all eigenvalues are equal to the noise variance $\lambda_{1}=\lambda_{2}=$ $\cdots=\lambda_{p}=\sigma_{\eta}^{2}$ [19]. For this reason $\left(\lambda_{p} / \lambda_{1}\right)=1$. Under the $H_{1}$ hypothesis, the eigenvalues of $\boldsymbol{R}_{\boldsymbol{x}}$ are equal to the sum of the noise variance and the variance of the primary user signal [17]. Hence, under the $H_{1}$ hypothesis, the eigenvalues are ordered as $\rho_{1}<\rho_{2} \ldots .<\rho_{p}$ and so $\left(\rho_{p} /\right.$ $\left.\rho_{1}\right)>\gamma$. For the MME, the test statistic is defined by the value $(T S)=\lambda_{p} / \lambda_{1}$ ( $\lambda_{1}$ is the minimum, $\lambda_{p}$ is the maximum eigenvalue) $[17,22] . \gamma_{m m e-c}$ represents the corrected threshold value, which is greater than 1 . Before we can obtain the threshold and $P_{f a}$ values for the MME, we will need an important theorem based on the random matrix theory. This theorem indicates that the deterministic values of the largest and smallest eigenvalues of the $\boldsymbol{R}_{\boldsymbol{\eta}}$ matrix are respectively $\lambda_{\max }=$ $\sigma_{\eta}^{2} / n(\sqrt{n}+\sqrt{p})^{2}$ and $\lambda_{\min }=\sigma_{\eta}^{2} / n(\sqrt{n}-\sqrt{p})^{2} \quad[15$, 16]. Then, if $P_{f a}$ threshold is obtained for MME, it is described as:

$P_{f a}=P\left(H_{1} \mid H_{0}\right)=P\left(\frac{\lambda_{\max }\left(\boldsymbol{R}_{\boldsymbol{\eta}}\right)}{\lambda_{\min }\left(\boldsymbol{R}_{\boldsymbol{\eta}}\right)}>\gamma_{m m e-c}\right)$

$P_{f a}=P\left(\lambda_{\max }\left(\boldsymbol{R}_{\boldsymbol{\eta}}\right)>\gamma_{m m e-c} \lambda_{\min }\left(\boldsymbol{R}_{\boldsymbol{\eta}}\right)\right)$

Since the probability distribution function of the largest eigenvalue here is known, one side of the equation must be likened to the Tracy-Widom distribution of order 1 . For this reason, if $\mu_{n, p, 0}$ and $\sigma_{c}$ coefficients are added to both sides of the equation, one side of the equation will converge to Tracy-Widom distribution of order 1 with $(n, p \rightarrow \infty)$ condition.

$P_{f a}=P\left(\lambda_{\max }\left(\boldsymbol{R}_{\boldsymbol{\eta}}\right) \frac{\sigma_{\eta}^{2}}{n} \frac{n}{\sigma_{\eta}^{2}}>\gamma_{m m e-c} \frac{\sigma_{\eta}^{2}}{n}(\sqrt{n}-\sqrt{p})^{2}\right)$

Using the survival function $[25,26]$, if the equation is arranged, the following is obtained

$P_{f a}=P\left(\lambda_{\max }\left(\boldsymbol{R}_{\boldsymbol{\eta}}\right) \frac{n}{\sigma_{\eta}^{2}}>\gamma_{m m e-c}(\sqrt{n}-\sqrt{p})^{2}\right)$

Then, $P_{f a}$ is defined as

$P_{f a}=P\left(\lambda_{\max }(\boldsymbol{A}(n))>\gamma_{m m e-c}(\sqrt{n}-\sqrt{p})^{2}\right)$
Since the probability distribution function of the largest eigenvalue here is known, if $\mu_{n, p, 0}$ and $\sigma_{c}$ coefficients are added to both sides of the equation, one side of the equation will converge to Tracy-Widom distribution of order 1 with $(n, p \rightarrow \infty)$ condition (see Eq. (2)).

$P_{f a}=$

$\left(\left(\frac{\lambda_{\max } A(n)-\mu_{n, p, 0}}{\sigma_{c}}\right)>\gamma_{m m e-c}\left(\frac{(\sqrt{n}-\sqrt{p})^{2}-\mu_{n, p, 0}}{\sigma_{c}}\right)\right)$

Using the survival function $[25,26]$, if the equation is arranged, the following is obtained.

$P_{f a}=1-F_{1}\left(\gamma_{m m e-c} \frac{(\sqrt{n}-\sqrt{p})^{2}-\mu_{n, p, 0}}{\sigma_{c}}\right)$

$\gamma_{m m e-c}$ indicates the recommended and corrected threshold value.

$F_{1}^{-1}\left(1-P_{f a}\right)=\left(\gamma_{m m e-c} \frac{(\sqrt{n}-\sqrt{p})^{2}-\mu_{n, p, 0}}{\sigma_{c}}\right)$

If $\mu_{n, p, 0}$ and $\sigma_{c}$ values are substituted

$F_{1}^{-1}\left(1-P_{f a}\right)=\left(\gamma_{m m e-c} \frac{(\sqrt{n}-\sqrt{p})^{2}-\left(\sqrt{n-\frac{1}{2}}+\sqrt{p-\frac{1}{2}}\right)^{2}}{\sqrt{\frac{n p}{2+n p}\left(\sigma_{n, p, 0}^{2}-\mu_{n, p, 0}^{2}\right)}}\right)$

Finally $\gamma_{m m e-c}$ becomes

$\gamma_{m m e-c}=$

$F_{1}^{-1}\left(1-P_{f a}\right) \cdot\left(\frac{\sqrt{\frac{n p}{2+n p}\left(\sigma_{n, p, 0}^{2}-\mu_{n, p, 0}^{2}\right)}}{(\sqrt{n}-\sqrt{p})^{2}+(\sqrt{n-1 / 2}+\sqrt{p-1 / 2})^{2}}\right)$

where $F_{1}$ shows Tracy-Widom cumulative distribution function of order 1. This distribution function is defined as

$F_{1}(t)=\exp \left(\int_{t}^{\infty}\left(q(u)+(u-t) q^{2}(u)\right) \mathrm{d} u\right)$.

where, $q(u)$ is the solution of the nonlinear painleve II differential equation and is described as:

$q^{\prime \prime}(u)=u q(u)+2 q^{3}(u)$

The specific values for this function are given in Tab. 1 [16].

Table 1 Some Numerical values for the Tracy-Widom distribution

\begin{tabular}{|c|c|c|c|c|c|c|c|c|c|c|c|}
\hline$x$ & -3.90 & -3.18 & -2.78 & -1.91 & -1.27 & -0.59 & 0.45 & 0.98 & 2.02 \\
\hline$F_{1}(x)$ & 0.01 & 0.05 & 0.10 & 0.30 & 0.50 & 0.70 & 0.90 & 0.95 & 0.99 \\
\hline
\end{tabular}

\subsection{Derivation of $P_{d}$ for MME:}

In this case, the $H_{1}$ hypothesis now applies. The sample covariance matrix of the received signal is not 102 is hart matrix anymore and $\boldsymbol{R}_{\boldsymbol{\eta}}$ turns into $\boldsymbol{R}_{\boldsymbol{x}}$. Thereby the smallest and largest eigenvalues of the $\boldsymbol{R}_{\boldsymbol{x}}$ are defined as follows: 
$\lambda_{\max }\left(\boldsymbol{R}_{\boldsymbol{x}}(n)\right) \approx \rho_{p}+\lambda_{\max }\left(\boldsymbol{R}_{\boldsymbol{\eta}}\right)$

$\lambda_{\min }\left(\boldsymbol{R}_{\boldsymbol{x}}(n)\right) \approx \rho_{1}+\sigma_{\eta}^{2}$.

where $\rho_{p} \quad$ and $\quad \rho_{1}$ indicates $\lambda_{\max }\left(\boldsymbol{R}_{x}(n)\right)$ and $\lambda_{\min }\left(\boldsymbol{R}_{\boldsymbol{x}}(n)\right)$ respectively.

Then, $P_{d}$ for MME;

$P_{d}=P\left(H_{1} \mid H_{1}\right)=P\left(\frac{\lambda_{\max }\left(\boldsymbol{R}_{x}\right)}{\lambda_{\min }\left(\boldsymbol{R}_{x}\right)}>\gamma_{m m e-c}\right)$

$P_{d}=P\left(\lambda_{\max }\left(\boldsymbol{R}_{x}\right)>\gamma_{m m e-c} \lambda_{\min }\left(\boldsymbol{R}_{x}\right)\right)$

If the values of $\lambda_{\max }\left(\boldsymbol{R}_{\boldsymbol{x}}(n)\right)$ and $\lambda_{\min }\left(\boldsymbol{R}_{\boldsymbol{x}}(n)\right)$ are substituted in place, Eq. (24) is obtained.

$P_{d}=P\left(\lambda_{\max }\left(\boldsymbol{R}_{\boldsymbol{\eta}}(n)\right)\right)>\gamma_{m m e-c}\left(\rho_{1}+\sigma_{\eta}^{2}\right)-\rho_{p}$

One side of the equation is likened to the Tracy-Widom distribution of order 1

$P_{d} \approx$

$P\left(\frac{\left(\lambda_{\max }\left(\boldsymbol{R}_{\boldsymbol{\eta}}(n)\right)-\mu_{n, p, 0}\right)}{\sigma_{c}}\right)>\frac{\left(\gamma_{m m e-c}\left(\rho_{1}+\sigma_{\eta}^{2}\right)-\rho_{p}\right)-\mu_{n, p, 0}}{\sigma_{c}}$

Finally, $P_{d}$ equation is obtained by using survival function

$P_{d} \approx$

$1-F\left(\frac{\left(\gamma_{m m e-c}\left(\rho_{1}+\sigma_{\eta}^{2}\right)-\rho_{p}\right)-n^{-1}\left(\sqrt{n-\frac{1}{2}}+\sqrt{p-\frac{1}{2}}\right)^{2}}{\sqrt{\frac{n p}{2+n p}\left(\sigma_{n, p, 0}^{2}-\mu_{n, p, 0}^{2}\right)}}\right)$

In view of Eq. (26), the $P_{d}$ value is not related to the noise power as opposed to the ED. The $P_{d}$ value depends only on the $\gamma$ value with $n$ and $p$ values of signal and noise. For this reason, these methods are also defined as blind spectrum sensing.

\subsection{Derivation of $P_{f a}$ and Threshold for the MET:}

For MET method, it has been identified as TS $=\lambda_{\max }\left(\boldsymbol{R}_{\boldsymbol{\eta}}(n)\right) / \operatorname{Trace}\left(\boldsymbol{R}_{\boldsymbol{\eta}}(n)\right)$. If $P_{f a}$ is calculated in the method;

$P_{f a}=P\left(\frac{\lambda_{\max }\left(\boldsymbol{R}_{\boldsymbol{\eta}}(n)\right)}{\operatorname{Trace}\left(\boldsymbol{R}_{\boldsymbol{\eta}}(n)\right)}>\gamma_{\text {met }-c}\right)$

$P_{f a}=P\left(\lambda_{\max }\left(\boldsymbol{R}_{\boldsymbol{\eta}}(n)\right)>\gamma_{\text {met }-c} \operatorname{Trace}\left(\boldsymbol{R}_{\boldsymbol{\eta}}(n)\right)\right)$

$P_{f a}=$

$P\left(\frac{\lambda_{\max }\left(\boldsymbol{R}_{\boldsymbol{\eta}}(n)\right)-\mu_{n, p, 0}}{\sigma_{c}}>\frac{\gamma_{\operatorname{met}-c} \operatorname{Trace}\left(\boldsymbol{R}_{\boldsymbol{\eta}}(n)\right)-\mu_{n, p, 0}}{\sigma_{c}}\right)$

Finally, $P_{f a}$ equation becomes as in (32).

$P_{f a}=1-F_{1}\left(\gamma_{m e t-c} \frac{\operatorname{Trace}\left(\boldsymbol{R}_{\boldsymbol{\eta}}(n)\right)-\mu_{n, p, 0}}{\sigma_{c}}\right)$
$F_{1}^{-1}\left(1-P_{f a}\right)=\left(\gamma_{m e t-c} \frac{\operatorname{Trace}\left(\boldsymbol{R}_{\boldsymbol{\eta}}(n)\right)-\mu_{n, p, 0}}{\sigma_{c}}\right)$

Then finally, $\gamma_{m e t-c}$ described as follows.

$\gamma_{m e t-c}=F_{1}^{-1}\left(1-P_{f a}\right)\left(\frac{\operatorname{Trace}\left(\boldsymbol{R}_{\boldsymbol{\eta}}(n)\right)-\mu_{n, p, 0}}{\sigma_{c}}\right)$

4.3 Derivation of $P_{d}$ for the MET:

$P_{d}=P\left(\frac{\lambda_{\max }\left(\boldsymbol{R}_{x}(n)\right)}{\operatorname{Trace}\left(\boldsymbol{R}_{x}(n)\right)}>\gamma_{\text {met }-c}\right)$

$P_{d}=1-F_{1}\left(\frac{\gamma_{m e t-c} \operatorname{Trace}\left(\boldsymbol{R}_{x}(n)\right)-\rho_{p}-\mu_{n, p, 0}}{\sigma_{c}}\right)$

\subsection{Derivation of $P_{f a}$ and threshold for the ME-GM}

In this method, again, while all eigenvalues are equal to 1 in the $H_{0}$ hypothesis, this equation should be greater than 1 since it will be $\lambda_{\max }>\lambda_{\min }$ in the $H_{I}$ hypothesis. In the corresponding method it is defined as $T S=\lambda_{\max }\left(\boldsymbol{R}_{\boldsymbol{\eta}}(n) /\left(\prod_{i=1}^{p} \lambda_{i}\right)^{1 / p}[15]\right.$.

$P_{f a}=P\left(\lambda_{\max }\left(\boldsymbol{R}_{\boldsymbol{\eta}}(n)\right)>\gamma_{m e-g m-c}\left(\prod_{i=1}^{p} \lambda_{i}\right)^{1 / p}\right)(35)$

In this equation, as it will be $\left(\prod_{i=1}^{p} \lambda_{i}\right)^{1 / p} \approx 1$ for the hypothesis $H_{0}[15]$ :

$P_{f a}=P\left(\frac{\lambda_{\max }\left(\boldsymbol{R}_{\boldsymbol{\eta}}(n)\right)-\mu_{n, p, 0}}{\sigma_{C}}>\frac{\gamma_{m e-g m-c}-\mu_{n, p, 0}}{\sigma_{C}}\right)$

the above is obtained, and finally $P_{f a}$ and threshold are obtained as in Eqs. (37) and (38).

$P_{f a}=1-F_{1}\left(\frac{\gamma_{m e-g m-c}-\mu_{n, p, 0}}{\sigma_{C}}\right)$

$\gamma_{m e-g m-c}=F_{1}^{-1}\left(1-P_{f a}\right) \sigma_{C}+\mu_{n, p, 0}$

\subsection{Derivation of $P_{d}$ for the ME-GM}

$P_{d}=P\left(\left(\lambda_{\max }\left(\boldsymbol{R}_{x}(n)\right)>\gamma_{m e-g m}\left(\prod_{i=1}^{p} \lambda_{i}\right)^{1 / p}\right)\right.$

The above equation could be written [15]. If $\lambda_{\max }$ is written in place using Eq. (20)

$P_{d}=P\left(\rho_{1}+\lambda_{\max }\left(\boldsymbol{R}_{\boldsymbol{\eta}}(n)\right)>\gamma_{m e-g m}\left(\prod_{i=1}^{p} \lambda_{i}\right)^{1 / p}\right)$

the above equation is obtained and again $\mu_{n, p, 0}$ and $\sigma_{c}$ are added and edited on both sides of the equation, $P_{d}$ is found as in Eq. (41).

$P_{d} \approx 1-F_{1}\left(\frac{\left(\gamma_{m e-g m}\left(\Pi_{i=1}^{p} \lambda_{i}\right)^{1 / p}-\rho_{1}\right)-\mu_{n, p, 0}}{\sigma_{C}}\right)$

If $\gamma_{m e t-c}$ 


\section{SIMULATION}

The theoretical analyses have been simulated in the MATLAB environment. $P_{f a}, 802.22$ was selected as the value set by the working group $\left(P_{f a}=0.1\right)$. By selecting the number of antennas in the secondary user $(p=6)$, BPSK and 1bit / symbol ratio were used. The primary user signal was randomly generated with the rand command. In the simulations, the classical and proposed methods for MME, MET and ME-GM are shown for Weibull channel simulation. To see the effect of noise uncertainty factor on algorithm performance, this value has been chosen at two different values [16]. Algorithms for Monte Carlo simulation have been run 1000 times.

In the traditional energy sensing method, noise power needs to be known. In practice, the estimated noise power may be different from the actual noise power $[9,15]$. For this reason, some uncertainties are in question at the calculation of the noise power. Assuming the estimated noise power is $\tilde{\sigma}_{\eta}^{2}=\alpha \sigma_{\eta}^{2}$, the noise uncertainty factor is defined as:

$$
B=\max \left\{10 \log _{10} \alpha\right\} \mathrm{dB}
$$

In practice, the noise uncertainty factor at the receiver can normally range from $1 \mathrm{~dB}$ to $2 \mathrm{~dB}[10,11]$. For this reason the simulations are carried out under different noise uncertainty conditions. For a more accurate interpretation of the results in Fig. 3, the ED method is also shown in the graph. SNR has been used as given in the relevant part of the source [16], where the coefficients $m$ and $k$ are the scale and shape parameters for the Weibull distribution, respectively [27].

When the graph is analysed, the limit has been increased to $-15 \mathrm{~dB}$ with the proposed method so that the detection probability could become 1 while the classical method has a noise limit of $-12 \mathrm{~dB}$ (for $0 \mathrm{~dB}$ noise uncertainty). The proposed method is more successful than the classical method even in the case of $2 \mathrm{~dB}$ noise uncertainty.

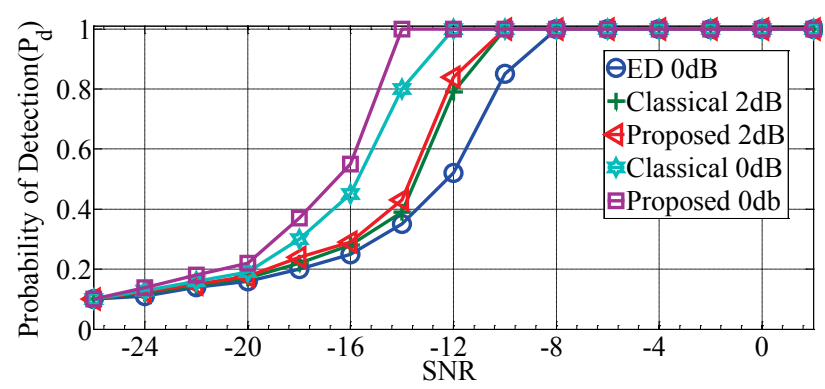

Figure 3 Weibull channel simulation results for MME: $n=1000, p=6, P_{f a}=$ $0.1, m=3.5, k=3.5$ (under different noise uncertainty conditions)

Figs. 4 and 5 show the $P_{d}-S N R$ results for MET and ME-GM methods, respectively. When Fig. 4 is analysed, it seems that MME is more successful under the specified conditions. Obviously, the proposed method has improved performance in all cases. As it could be seen in the figure, ED is more successful than MET under noise uncertainty of $0 \mathrm{~dB}$. When the proposed equations are used, ME-GM seems to be more successful than ED for difference noise uncertainty conditions.

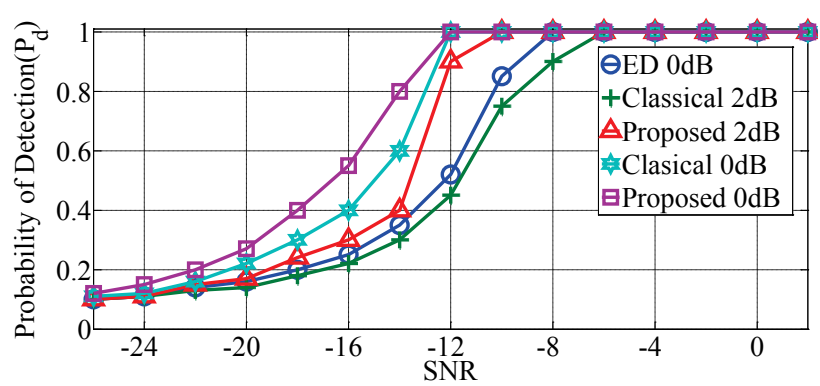

Figure 4 Weibull channel simulation results for MET: $n=1000, p=6, P_{f a}=$ $0.1, m=3.5, k=3.5$ (under different noise uncertainty conditions)

It is also seen that MME is more successful than MET in all cases when Fig. 3 and Fig. 4 are interpreted together. When the proposed probability distribution is used, the MME has reached a detection probability of even $-14 \mathrm{~dB}$. But this value for MET is $-12 \mathrm{~dB}$. The proposed method also provided a greater performance gain for MET than MME.

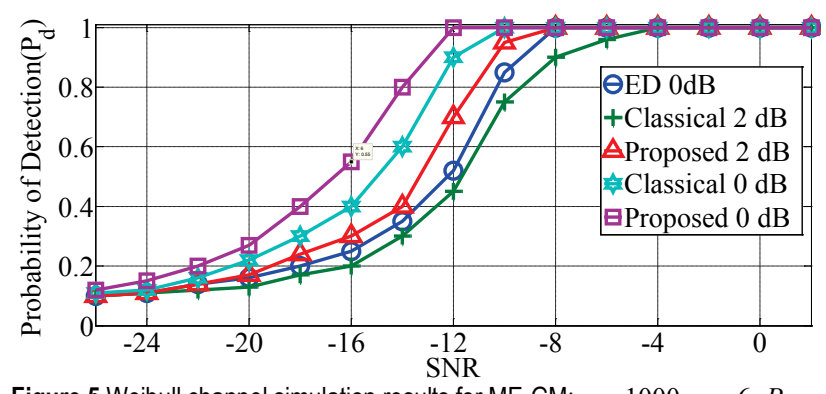

Figure 5 Weibull channel simulation results for ME-GM: $n=1000, p=6, P_{f a}=$ $0.1, m=3.5, k=3.5$ (under different noise uncertainty conditions)

In addition to these MET shows almost the same performance as ME-GM but ME-GM is more advantageous in terms of calculation cost (see Eq. (32) and (38)). This situation may be the cause of choice for ME-GM.

To investigate the improvement in $P_{d}$ for the proposed scheme when the sample size is decreased, we varied $n$ to 500 and 2000 samples. The results are plotted in Fig. 6. (for $n=1000$, see previous charts). When the graph is analysed, it is seen that the proposed method positively contributes to the results, especially with a more significant improvement for 500 samples numbers. For the probability of detection to be 1 in classical methods in 500 sample numbers while there is a noise threshold of $5 \mathrm{~dB}$, this value reaches a value of $0 \mathrm{~dB}$ with the proposed method.

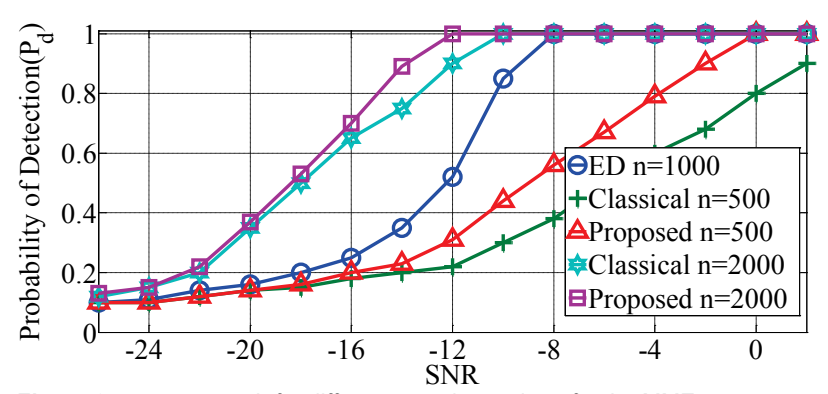

Figure $6 S N R-P_{d}$ graph for different sample numbers for the MME: $n=1000$, $p=6, m=3.5, k=3.5$

The reason for this increase in performance is that the resulting $L_{c}$ statistic correctly expresses the largest 
eigenvalue probability distribution. Because in the detection theory, the distribution functions of the sensing parameters and the centre, variance values of these functions are the factors that directly affect the detection performance.

Fig. 7 shows ROC analysis with the proposed variance and scale adjustment. When the ROC curves are analysed, it could be seen that the proposed method shows improvement in both algorithms and MME is the most successful method. SNR ratio was selected to be $-15 \mathrm{~dB}$ when performing roc analysis. Since MET and ME-GM represents similar performances, they are not included in the roc analysis. As seen in the graphic, MME also showed less improvement compared to ME-GM.

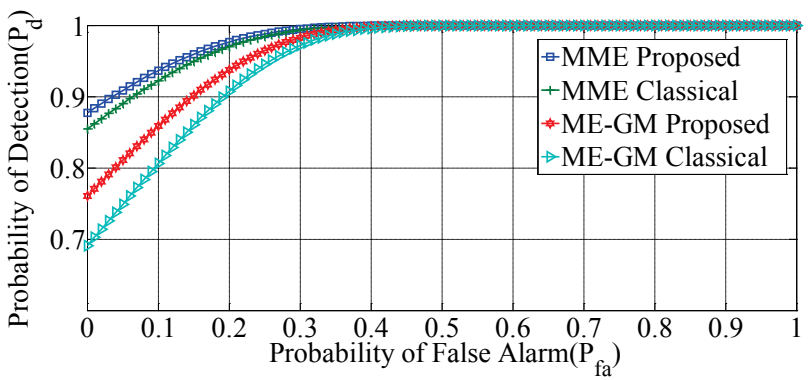

Figure 7 ROC analyses for MME and MET: $n=1000, p=6, m=3.5, k=3.5$, $S N R=-15 \mathrm{~dB}$, Noise Uncertainty $=0 \mathrm{~dB}$

\section{DISCUSSION}

There are two fundamental problems in spectrum sensing: cognitive radio and radar systems. The first is the most successful detection despite the high noise ratio. The second is detected as soon as possible. For a successful detection based on the theory of detection, the parameters of the method must be expressed correctly. We have considered the performance problems resulting from the fact that the function that expresses the largest eigenvalue distribution in the covariance / eigenvalue spectrum detection methods up to now cannot express this distribution correctly. These parameters must be expressed with closed functions in order to obtain the threshold value in these methods. The studentize eigenvalue distribution approach clearly shows the distribution as shown in Fig. 2. As seen from the graphs when using the corrected threshold values, we have improved two important factors for the theory of detection.

\section{CONCLUSION}

In this study, it is aimed to develop covariance/ eigenvalue based spectrum detection methods. It is necessary to use the probability distribution of the covariance matrix eigenvalues to obtain the threshold value in these methods. We have recovered the threshold values using the studentize distribution function instead of the probability distribution function used up to now. Thus, the largest eigenvalue distribution is used by being studentized for MME, MET and ME-GM methods and the probability of detection and threshold values has been regained. It is seen that the proposed method gives results that are more successful especially at low sample lengths in simulations so that the proposed method can perform the spectrum sensing process in a shorter time. Because the Weibull fading channel is the best simulated wireless communication channel, simulations are applied in Weibull fading channel and compared with the ED method under different noise uncertainty conditions. The proposed method provided a remarkable performance boost especially in the case of limited sample lengths.

\section{REFERENCES}

[1] Jayaweera, S. K. (2014). Signal Processing for Cognitive Radios. John Wiley \& Sons, Inc. Melbourne. https://doi.org/10.1002/9781118824818

[2] Mitola, J. \& Maguire, Jr. G. Q. (1999). Cognitive Radio: making Software Radios More. IEEE Pers. Commun., 6(4), 1318. https://doi.org/10.1109/98.788210

[3] Shah, H. A. \& Usman, M. (2015). Bioinformatics-inspired Quantized Hard Combination-based Abnormality Detection for Cooperative Spectrum Sensing in Cognitive Radio Networks. IEEE Sensors J., 15(4), 2324-2334. https://doi.org/10.1109/JSEN.2014.2375363

[4] Devroye, N., Vu, M. \& Tarokh, V. (2008). Cognitive radio networks. IEEE Signal Process., 25(6), 12. https://doi.org/10.1109/MSP.2008.929286

[5] Cabric, D. (2008). Addressing the feasibility of cognitive radios. IEEE Signal Process. Mag., 25(6), 85-93. https://doi.org/10.1109/MSP.2008.929367

[6] Cabric, D., Mishra, S. M. \& Brodersen, R. W. (2004). Implementation issues in spectrum sensing for cognitive radios. Proc. Asilomar Conf. Signals Systems Computers, 1, 772-776. https://doi.org/10.1109/ACSSC.2004.1399240

[7] Zeng, Y., Liang, Y. -C., Hoang, A. T. \& Zhang, R. (2010). A review on spectrum sensing for cognitive radio: challenges and solutions. EURASIP J. Adv. Signal Process. 2010(2), 115. https://doi.org/10.1155/2010/381465

[8] Chen, H. S., Gao, W. \& Daut, D. G. (2007). Signature Based Spectrum Sensing Algorithms for IEEE 802.22 WRAN. Proc. IEEE Intern. Conf. Commun. / Glasgow, 6487-6492. https://doi.org/10.1109/ICC.2007.1073

[9] Digham, F. F., Alouini, M. S. \& Simon, M. K. (2007). On the Energy Detection of Unknown Signals over Fading Channels. IEEE Trans. Commun., 55(1), 21-24. https://doi.org/10.1109/TCOMM.2006.887483

[10] Urkowitz, H. (1967). Energy Detection of Unknown Deterministic Signals. Proc. IEEE, 55(4), 523-531. https://doi.org/10.1109/PROC.1967.5573

[11] Gardner, W. A. (1991). Exploitation of Spectral Redundancy in Cyclostationary Signals. IEEE Signal Processing Mag. 8, 14-36. https://doi.org/10.1109/79.81007

[12] Gardner, W. A. (1987). Spectral Correlation of Modulated Signals: Part I-analog Modulation. IEEE Trans. Commun., 35(6), 584-595. https://doi.org/10.1109/TCOM.1987.1096820

[13] Gardner, W. A., Brown, W. A. \& Chen C. K. (1987). Spectral Correlation of Modulated Signalspart II: Digital Modulation. IEEE Trans. Commun., 35(6), 595-601. https://doi.org/10.1109/TCOM.1987.1096816

[14] Han, N., Shon, S. H., Joo, J. O. \& Kim, J. M. (2006). Spectral Correlation Based Signal Detection Method for Spectrum Sensing in IEEE 802.22 WRAN systems. Proc. Intern. Conf. Advanced Commun. Technology. / Korea, 591-601.

[15] Pillay, N. \& Xu, H. J. (2011). Blind Eigenvalue-Based Spectrum Sensing for Cognitive Radio Networks. IET Comm. 6(11), 1388-1396. https://doi.org/10.1049/iet-com.2011.0506

[16] Zeng, Y. \& Liang, Y. C. (2009). Eigenvalue-Based Spectrum Sensing Algorithms for Cognitive Radio. IEEE Transactions on Communications, 57(6), 1784-1793. https://doi.org/10.1109/TCOMM.2009.06.070402 
[17] Zeng, Y. \& Liang, Y. C. (2009). Spectrum-Sensing Algorithms for Cognitive Radio Based on Statistical Covariances. IEEE Trans. Veh. Technol., 58(4), 1804-1815. https://doi.org/10.1109/TVT.2008.2005267

[18] Charan, C. \& Pandey, R. (2016). Eigenvalue Based Double Threshold Spectrum Sensing Under Noise Uncertainty for Cognitive Radio. Optik - International Journal for Light and Electron Optics, 127(15), 5968-5975. https://doi.org/10.1016/j.ijleo.2016.04.049

[19] Johnstone, I. (2001). On the Distribution of the Largest Eigenvalue in Principal Components Analysis. Ann. Statist., 29(2), 295-327. https://doi.org/10.1214/aos/1009210544

[20] Jin, M., Guo, Q., Xi, J., Youming Li, Y., Yu, Y. \& Huang, D. (2015). Spectrum Sensing Using Weighted Covariance Matrix in Rayleigh Fading Channels. IEEE Transactions on Vehicular Technology, 64(11), 5137-5148. https://doi.org/10.1109/TVT.2014.2379924

[21] Ma, Z. (2012). Accuracy of the Tracy-Widom Limits for the Extreme Eigenvalues in White Wishart Matrices. International Statistical Institute/Bernoulli Society, 18(1), 322-359. https://doi.org/10.3150/10-BEJ334

[22] Nadler, B. (2011). On the distribution of the Ratio of the Largest Eigenvalue to the Trace of a Wishart Matrix. $J$. Multivariate Anal., 102, 363-371. https://doi.org/10.1016/j.jmva.2010.10.005

[23] Deo, S. R. (2016). On the Tracy-Widom Approximation of Studentized Extreme Eigenvalues of Wishart Matrices. Journal of Multivariate Analysis, 147, 265. https://doi.org/10.1016/j.jmva.2016.01.010

[24] Kortun, A., Sellathurai, M., Ratnarajah, T. \& Zhong, C. (2012). Distribution of the Ratio of the Largest Eigenvalue to the Trace of Complex Wishart Matrices. IEEE Transactions on Signal Processing, 60(10). https://doi.org/10.1109/TSP.2012.2205922

[25] Soshnikov, A. (2002). A note on universality of the distribution of the largest eigenvalues in certain sample covariance matrices. J. Stat. Phys., 108, 1033-1056. https://doi.org/10.1023/A:1019739414239

[26] Kleinbaum, D. G. \& Mitchel, K. (2012). Survival analysis: A Self-learning text (Third ed.), Springer, New York. https://doi.org/10.1007/978-1-4419-6646-9

[27] Crowder, M. (1989). A Multivariate Distribution with Weibull Connections. Royal Statist. Soc. J., 51, 93-107.

[28] Tiwari, K. \& Saini, D. S. (2015). SER improvisation of MIMO-MRC system over Weibull-Gamma fading channel. Proc. of the International Conference on Signal Processing and Communication (ICSC'2015). / Hannover, 70-73. https://doi.org/10.1109/ICSPCom.2015.7150622

[29] Bradley, A. P. (1997). The use of the Area under the ROC Curve in the Evaluation of Machine Learning Algorithms. Pattern Recogn., 30(7), 1145-1159. https://doi.org/10.1016/S0031-3203(96)00142-2

[30] Althouse, D. A. (2015). Statistical graphics in action: making better sense of the ROC curve. International Journal of Cardiology, 215, 9-10. https://doi.org/10.1016/j.ijcard.2016.04.026

\section{Contact information:}

\section{Cebrail ÇIFLIKL}

Erciyes University, Vocational High School,

Electronic and Automation Department,

Kayseri, Turkey

cebrailc@erciyes.edu.tr

\section{Fatih Yavuz ILGIN}

(Corresponding Author)

Erzincan University, Vocational High School,

Electronic and Automation Department

Fatih Mahallesi, 726. Sk., 24100 Merkez/Erzincan, Turkey

E-mail: fyilgin@erzincan.edu.tr 